Check for updates

\title{
Subgroup Analysis of Topical Tranexamic Acid in Primary Total Hip Arthroplasty
}

Walter A. Anazonwu, BS*; John R. Tuttle, MD*; Lee E. Rubin, MD*

\section{Introduction}

Intraarticular or "topical" tranexamic acid (TXA) has increasingly received attention for reducing blood loss following total joint arthroplasty $[1,3,6,8,14]$. While our institution has seen transfusion rates drop from $17.5 \%$ to $5.5 \%$ after administration of topical TXA in total joint replacement, it is still not known which patients will benefit most from TXA administration [14]. Patients undergoing total hip arthroplasty (THA) at our institution continue to have a higher allogeneic transfusion rate compared to total knee arthroplasty (TKA). While THA patients respond to topical TXA, the question remains as to which specific subset of THA patients might benefit most from administration of topical TXA. To answer this question we performed a retrospective cohort study that involved 123 THA patients who received topical TXA, and compared them to 111 controls who did not receive TXA treatment. These patients were subdivided into groups based on gender, age, BMI, preoperative hemoglobin, and surgical approach.

Our goal in this investigation is to identify characteristics that will more accurately justify the use of topical TXA in THA; the ultimate goal is for a surgeon to correctly identify patients preoperatively (prospectively) who will most consistently benefit from topical TXA administration. Preoperative identification of patients who would most likely benefit from topical TXA administration would allow for more targeted use of the drug, ideally reducing cost and unnecessary exposure.

\section{Methods}

Following IRB approval, we retrospectively reviewed 234 primary hip arthroplasties performed by 5 orthope- dic surgeons at a single institution between March 2012 and March 2013. Treatment with topical TXA in all primary hip patients was initiated intraoperatively starting September 1st, 2012. The months of August and September of 2012 were excluded from the study in order to prevent any overlap of the experimental and control group. Bilateral and revision hips were excluded from this study.

All patients received spinal or general anesthesia as well as local anesthesia; 10cc of $0.5 \%$ Marcaine without epinephrine was used at the operative site after wound closure. Patients received preoperative antibiotics within 1 hour of surgical incision. Antibiotics used included: cefazolin, vancomycin (if MRSA history was present), or Clindamycin (if significant cephalosporin allergy was observed). Standard postoperative DVT prophylaxis was used by all of the surgeons that participated in the study (e.g. TEDS, SCDs, and chemical prophylaxis). One surgeon used postoperative aspirin for chemical DVT prophylaxis, while the other four used Coumadin. No intraoperative drains were placed. One gram of TXA was injected in the pericapsular and deep tissue spaces, or intra-articularly following iliotibial band closure, depending on the surgeon's preference. Otherwise, no changes were made to each surgeon's individual surgical and postoperative protocols between the control and experimental groups. No primary, unilateral total joint patients were excluded from TXA use.

Transfusion was triggered by hemoglobin of less than 8 $\mathrm{g} / \mathrm{dL}$ or symptomatic anemia for all patients in both control and experimental groups. Each chart was reviewed via the electronic medical record and the following variables were

* Warren Alpert Medical School of Brown University, Providence, RI, USA

(C) 2015 Walter A. Anazonwu, John R. Tuttle, Lee E. Rubin. All rights reserved DOI: $10.15438 /$ rr. $5.1 .80 \bullet$ ISSN 2331-2262 (print) • ISSN 2331-2270 (online)

For complete copyright and licensing information please refer to the end of this article. 
recorded for analysis: age, gender, BMI, transfusions, preoperative hemoglobin, postoperative hemoglobin, days in hospital, disposition, 30 day readmission, and complications (including UTI, MI, DVT, stroke, and death). No routine screening for DVT/PE was performed. Symptomatic DVT was confirmed by ultrasound.

Statistical analysis was used to confirm the significance of the results. The chi square test was used for discrete variables (e.g. transfusion rate and hospital disposition). Independent t-tests were used for continuous variables (e.g. drop in Hgb, BMI, and age). Statistical significance was defined as $\mathrm{P}<0.05$ (Table 2).

Table 1. Primary outcome

\begin{tabular}{|l|c|c|c|}
\hline & Before TXA & After TXA & P-value \\
\hline Readmission & 5 & 6 & 0.8928 \\
\hline Complications & 0 & 1 & 0.3411 \\
\hline Delta Hgb & $4.8+/-1.1$ & $4.0+/-1.0$ & $<0.0001$ \\
\hline Postoperative Hgb & $9.1+/-1.3$ & $9.8+/-1.4$ & 0.0001 \\
\hline Patients Transfused & 24 & 9 & 0.0016 \\
\hline Units Transfused & 39 & 13 & 0.0003 \\
\hline Length of Stay & $3.2+/-1.0$ & $3.1+/-1.0$ & 0.4362 \\
\hline
\end{tabular}

Data reported as mean +/-SD or total sum. $P$ values calculated using either independent T-test or chi square test.

Table 2. Demographic

\begin{tabular}{|l|c|c|c|}
\hline & $\begin{array}{c}\text { Before TXA }(\mathbf{N} \\
=\mathbf{1 1 1})\end{array}$ & $\begin{array}{c}\text { After TXA } \\
\mathbf{= 1 2 3})\end{array}$ & P-value \\
\hline Age & $63.3+/-13.5$ & $64.9+/-12.1$ & 0.3349 \\
\hline Male & 54 & 52 & 0.3282 \\
\hline Female & 57 & 71 & 0.3282 \\
\hline BMI & $30.3+/-5.2$ & $30.7+/-6.4$ & 0.6766 \\
\hline Preoperative Hgb & $13.9+/-1.4$ & $13.9+/-1.5$ & 0.8502 \\
\hline
\end{tabular}

Data reported as mean +/- SD or total sum. $P$ values calculated using either independent T-test or chi square test.

Table 3. Subgroup Population

\begin{tabular}{|l|c|c|}
\hline & Before TXA & After TXA \\
\hline Age $<50$ & 15 & 16 \\
\hline Age 50 to 65 & 50 & 46 \\
\hline Age $>65$ & 46 & 61 \\
\hline BMI $<30$ & 49 & 60 \\
\hline BMI $>30$ & 50 & 63 \\
\hline Female & 57 & 71 \\
\hline Male & 54 & 52 \\
\hline Hgb $<12$ & 6 & 10 \\
\hline Hgb $>12$ & 92 & 94 \\
\hline Anterior-lateral & 90 & 83 \\
\hline Anterior & 20 & 35 \\
\hline
\end{tabular}

Table 4. Primary outcomes within subgroups

\begin{tabular}{|c|c|c|c|}
\hline & Before TXA & After TXA & P-value \\
\hline \multicolumn{4}{|l|}{ Age $<50$} \\
\hline Transfusion & $2(13.3 \%)$ & $0(0.0 \%)$ & 0.13101 \\
\hline Delta Hgb & $4.7+/-0.8$ & $4.6+/-0.9$ & 0.7018 \\
\hline Postoperative Hgb & $9.4+/-1.2$ & $9.9+/-1.7$ & 0.3348 \\
\hline \multicolumn{4}{|l|}{ Age 50 to 65} \\
\hline Transfusion & $10(20.0 \%)$ & $3(6.5 \%)$ & 0.0538 \\
\hline Delta Hgb & $4.8+/-1.3$ & $4.2+/-1.0$ & 0.0192 \\
\hline Postoperative Hgb & $9.3+/-1.4$ & $10.1+/-1.4$ & 0.0105 \\
\hline \multicolumn{4}{|l|}{ Age $>65$} \\
\hline Transfusion & $12(26.1 \%)$ & $6(9.8 \%)$ & 0.0261 \\
\hline Delta Hgb & $4.8+/-0.9$ & $3.7+/-0.9$ & $<0.0001$ \\
\hline Postoperative Hgb & $8.9+/-1.3$ & $9.6+/-1.3$ & 0.0025 \\
\hline \multicolumn{4}{|l|}{$\mathrm{BMI}<30$} \\
\hline Transfusion & $11(22.4 \%)$ & $5(8.3 \%)$ & 0.0383 \\
\hline Delta Hgb & $4.6+/-1.1$ & $3.9+/-1.0$ & 0.0051 \\
\hline Postoperative Hgb & $9.2+/-1.4$ & $9.8+/-1.4$ & 0.023 \\
\hline \multicolumn{4}{|l|}{$\mathrm{BMI}>\mathbf{3 0}$} \\
\hline Transfusion & $12(24.0 \%)$ & $4(6.3 \%)$ & 0.0075 \\
\hline Delta Hgb & $4.8+/-1.1$ & $4.0+/-1.0$ & 0.0004 \\
\hline Postoperative Hgb & $9.1+/-1.3$ & $9.9+/-1.4$ & 0.0042 \\
\hline \multicolumn{4}{|l|}{ Female } \\
\hline Transfusion & $22(38.6 \%)$ & $8(11.3 \%)$ & 0.0003 \\
\hline Delta Hgb & $4.8+/-1.2$ & $4.1+/-1.0$ & 0.0005 \\
\hline Postoperative Hgb & $8.4+/-1.1$ & $9.3+/-1.1$ & 0.0001 \\
\hline \multicolumn{4}{|l|}{ Male } \\
\hline Transfusion & $2(3.7 \%)$ & $1(1.9 \%)$ & 0.5805 \\
\hline Delta Hgb & $4.7+/-1.1$ & $3.9+/-1.0$ & 0.0002 \\
\hline Postoperative Hgb & $9.9+/-1.1$ & $10.6+/-1.4$ & 0.0034 \\
\hline \multicolumn{4}{|l|}{$\mathrm{Hgb}<12$} \\
\hline Transfusion & $6(100 \%)$ & $3(30 \%)$ & 0.0063 \\
\hline Delta Hgb & $3.4+/-1.2$ & $3.0+/-0.7$ & 0.4451 \\
\hline Postoperative Hgb & $7.6+/-0.3$ & $7.8+/-0.5$ & 0.3126 \\
\hline \multicolumn{4}{|l|}{$\mathrm{Hgb}>12$} \\
\hline Transfusion & $16(17.4 \%)$ & $4(4.3 \%)$ & 0.0038 \\
\hline Delta Hgb & $4.9+/-1.1$ & $4.1+/-1.0$ & 0.0001 \\
\hline Postoperative Hgb & $9.2+/-1.3$ & $10.1+/-1.3$ & 0.0001 \\
\hline \multicolumn{4}{|l|}{ Anterior-Lateral } \\
\hline Transfusion & $19(21.1 \%)$ & $5(6.0 \%)$ & 0.00413 \\
\hline Delta Hgb & $4.4+/-1.9$ & $3.9+/-1.0$ & 0.0442 \\
\hline Postoperative Hgb & $9.2+/-1.4$ & $9.9+/-1.5$ & 0.0015 \\
\hline \multicolumn{4}{|l|}{ Anterior } \\
\hline Transfusion & $5(25.0 \%)$ & $2(5.7 \%)$ & 0.039 \\
\hline Delta Hgb & $5.5+/-1.1$ & $4.1+/-1.0$ & 0.0001 \\
\hline Postoperative Hgb & $8.7+/-1.1$ & $9.7+/-1.2$ & 0.0098 \\
\hline
\end{tabular}

Data reported as mean +/- SD or total sum. P values calculated using either independent T-test or chi square test. 


\section{Results}

All 234 THA were analyzed based on gender, age, BMI, preoperative hemoglobin, and surgical approach. Age was divided into: younger than 50 years, between 50 and 65 years, and older than 65 years, BMI was divided by obesity (defined as $>30$ by the World Health Organization). Preoperative hemoglobin $(\mathrm{Hgb})$ status was delineated by $12 \mathrm{~g} /$ $\mathrm{dL}$, and surgical approach was divided into direct anterior and anterolateral approach. There was no statistically significant difference in demographics between the pre and post TXA groups (Table 3 ). The total number of cases for each group is located in Table 1.

Topical TXA consistently reduced transfusion rate, increased postoperative $\mathrm{Hgb}$, and decreased the change in $\mathrm{Hgb}$ (Table 2). However, further analysis of the subgroups revealed that these effects were not evenly distributed (Table 4).

\section{GENDER}

Both males and females had a significant difference in their postoperative Hgb and delta Hgb. However, after administration of TXA, only females experienced a significant reduction in transfusion rate. The transfusion rate in females went from $38.6 \%$ to $11.3 \%$ after administration of TXA, $p=0.0003$. Males experienced a transfusion rate reduction from $3.7 \%$ to $1.9 \%$ after TXA administration, which was not significant ( $\mathrm{p}=0.5805)$. One possible explanation for the difference in transfusion rates between men and women could be the increased risk for transfusion normally seen in women who undergo THA [2]. As seen in the control group of this study, women generally have a lower average $\mathrm{Hgb}$ than men $(13.2 \mathrm{~g} / \mathrm{dL}$ compared to 14.6 $\mathrm{g} / \mathrm{dL}$ ), which results in women having lower postoperative $\mathrm{Hgb}(8.4 \mathrm{~g} / \mathrm{dL}$ compared to $9.9 \mathrm{~g} / \mathrm{dL})$. The female control group had 2 readmissions and no complications, while the TXA group had 3 readmissions and one UTI complication. The male control group had 3 readmissions and no complications, while the TXA group also had 3 readmissions and no complications.

\section{BODY MASS INDEX}

All patients, regardless of their BMI, experienced significant differences in their delta $\mathrm{Hgb}$, post-operative $\mathrm{Hgb}$, and transfusion rate. In patients with a BMI of $>30$, the control group had 3 readmissions and no complications, while the TXA group had 1 readmission and no complications. In patients with a BMI $<30$, the control group showed 1 readmission and no complications, while the TXA group had 5 readmissions and 1 UTI complication.

\section{AGE CATEGORY}

Patients younger than 50 years experienced no significant changes in delta $\mathrm{Hgb}$, postoperative $\mathrm{Hgb}$, and transfusion. In patients over 65 years and patients between 50 and 65 years, both groups had a significant difference in delta $\mathrm{Hgb}$ and postoperative $\mathrm{Hgb}$. Patients over 65 years experienced a significant reduction in transfusion rate $(26.1 \%$ to $9.8 \%$ after administration of TXA, $\mathrm{p}=0.0261$ ). Patients between 50 and 65 years experienced a transfusion reduction rate from $20 \%$ to $6.5 \%$ after administration of TXA, p $=0.0538$. In patients over 65 years, the control group had 2 readmissions and no complications, while the TXA group had 3 readmissions and 1 UTI complication. The control group of patients between 50 and 65 years contained 1 readmission and no complications, while the TXA group had 3 readmissions and no complications. The control group of patients less than 50 years contained no readmissions or complications, and the TXA group also had no readmissions or complications.

\section{PREOPERATIVE HEMOGLOBIN}

After TXA administration, patients with preoperative $\mathrm{Hgb}<12 \mathrm{~g} / \mathrm{dL}$ saw significant reductions in the rate of transfusion $(100 \%$ to $30 \%, \mathrm{p}=0.0063)$. Also, after TXA administration, patients with a preoperative $\mathrm{Hgb}$ of $>12 \mathrm{~g} /$ $\mathrm{dL}$ experienced both a significant reduction in the rate of transfusion $(17.4 \%$ to $4.3 \%, \mathrm{p}=0.0038)$, and a significant change in delta $\mathrm{Hgb}(4.9+/-1.1$ to $4.1+/-1.0, \mathrm{p}=0.0001)$. The control group in patients with a preoperative $\mathrm{Hgb}$ of $<$ $12 \mathrm{~g} / \mathrm{dL}$ had 1 readmission and no complications, and the TXA group also had 1 readmission and no complications. Additionally, in patients with a preoperative $\mathrm{Hgb}>12 \mathrm{~g} /$ $\mathrm{dL}$, the control group had 4 readmissions and no complications, while the TXA group had 3 readmissions and no complications.

\section{Surgical Approach to the Hip}

After administration with TXA, patients who underwent either the direct anterior approach (DAA) or anterior-lateral approach (AL) both experienced significant differences in their postoperative $\mathrm{Hgb}$, delta $\mathrm{Hgb}$, and transfusion rates. The control group in patients who underwent DAA had 3 readmissions and no complications, while the TXA group had 2 readmissions and no complications. Furthermore, in patients who underwent AL, the control group had 3 readmissions and no complications, while the TXA group had 2 readmissions and no complications. 


\section{Discussion}

While the perioperative administration of TXA is being used more widely in total joint replacement surgery, both the method / route of TXA application and the exact patient population who stands to benefit the most from TXA utilization in THA have yet to be established in the literature. The goal of this study was to retrospectively determine which patients undergoing THA had a significant response to topical TXA.

The greatest weakness of this study was its retrospective design. Patients were followed for 30 days postoperatively in the electronic database, and therefore long-term complications or complications managed at a different healthcare facility were not recorded in this study. Some subgroups may be under powered to determine a significant difference in our outcome variables. For example, no significant differences were found in the $<50$ age group; this may be a false negative, or younger patients may truly not benefit from topical TXA in THA.

This study is consistent with the current literature by revealing significant differences in transfusion rate reduction, delta Hgb, and postoperative Hgb with topical TXA $[7,12,13,15]$.

In concordance with Judge et al.'s paper, our study concludes that BMI has no bearing in primary hip replacement surgery despite TXA treatment [5]. The two BMI subgroups in our study showed no significant change in complication rates, and both subgroups experienced significant differences in their delta $\mathrm{Hgb}$, postoperative $\mathrm{Hgb}$, and transfusion rate after administration with TXA.

Surgical approach had no effect on the outcomes in THA despite TXA use. In both the direct anterior approach group and anterolateral approach group there was no significant change in complication rates. Also, both subgroups experienced significant differences in their delta $\mathrm{Hgb}$, postoperative $\mathrm{Hgb}$, and transfusion rate after administration with TXA.

Patients who are normally at risk for transfusions in THA appear to benefit the most with TXA treatment: women experienced a significant reduction in transfusion rate after TXA treatment. One explanation could be the increased risk for transfusion normally seen in women who undergo THA. According to Morrison et al.'s findings, the clinical significance of TXA is strongest in patients who have the highest anticipated blood loss [10]. Danninger et al. and Saleh et al. concluded that women are at an increased risk for transfusion in THA, our data is consistent with these findings showing a higher transfusion rate in women $(38.6 \%$ compared to $3.7 \%)$ [2,11]. Also, women had a relatively greater clinical response to TXA (with transfusion rate reduction from $38.6 \%$ to $11.3 \%$ in women compared to transfusion rate reduction from $3.7 \%$ to $1.9 \%$ in men).

According to Saleh et al., a risk factor for transfusion after THA is increased age [11].Our study has shown that the rate of transfusion was highest in patients over 65 years old (26\% compared to $20 \%$ in patients between 50 and 65 years old, and $13.3 \%$ in patients less than 50 years old). While the subgroup may be underpowered, patients less than 50 years of age do not appear to benefit from TXA use. This may be due to their ability to compensate for relative anemia compared to the older cohorts. Patients over the age of 65 consistently benefit from TXA use (Table 4).

Our study is consistent with the conclusion that low preoperative $\mathrm{Hgb}$ is associated with an increased risk of transfusion during admission for THA $[4,11] .100 \%$ of patients with $\mathrm{Hgb}<12.0 \mathrm{~g} / \mathrm{dL}$ received transfusion prior to TXA administration, while $17.4 \%$ of patients with $\mathrm{Hgb}>12.0 \mathrm{~g} /$ $\mathrm{dL}$ received transfusion prior to TXA administration. Only patients with $\mathrm{Hgb}>12.0 \mathrm{~g} / \mathrm{dL}$ experienced a significant change in delta and postoperative Hgb after TXA administration. There are two likely possibilities for this difference. The first is that patients with a low preoperative $\mathrm{Hgb}$ are more likely to receive an intraoperative transfusion which would alter both delta $\mathrm{Hgb}$ and postoperative $\mathrm{Hgb}$. Second, the number of patients in the $<12 \mathrm{Hgb}$ group may be too low to detect these differences. TXA appears to be effective despite preoperative Hgb status.

According to Mayr et al., when compared to the traditional AL approach, patients who undergo DAA experience a faster return to normal function [9]. Our results do not indicate a significant difference between the two approaches for THA regarding short term outcomes, and demonstrated similar blood product utilization in both groups. Patients undergoing either approach stand to benefit from TXA administration (Table 4).

\section{Conclusion}

According to this study, there are no restrictions on the use of topical TXA in THA, however not all patients should be expected to benefit equally. A preoperative $\mathrm{Hgb}$ $>12$ is protective against perioperative transfusions especially in combination with TXA, however TXA significantly reduces transfusion rates regardless of preoperative $\mathrm{Hgb}$ status. Female patients and those over 65 years of age appear to have the most reliable and consistent response to topical TXA use in THA. 


\section{References}

1. Chang CH, Chang Y, Chen DW, Ueng SWN, Lee MS. Topical Tranexamic Acid reduces blood loss and transfusion rates Associated with Primary Total Hip Arthroplasty. Clinical Orthopaedics and Related Research. 2014.

2. Danninger T, Rasul R, Poeran J, Stundner O, Mazumdar M, Fleischut PM, Poultsides L, Memtosoudis SG. Blood Transfusions in Total Hip and Knee Arthroplasty: an analysis of outcomes. ScientificWorld Journal. 2014.

3. Gilbody J, Dhotar HS, Perruccio AV, Davey JR. Topical tranexamic Acid reduces transfusion rates in total hip and knee arthroplasty. J Arthroplasty. 2014;29(4): 681-684.

4. Jans O, Jorgensen C, Kehlet H, Johansson PI. Role of Preoperative Anemia for Risk of Transfusion and Postoperative Morbidity in Fast-Track Hip and Knee Arthroplasty. Transfusion. 2014;54(3): 717-726.

5. Judge A, Batra RN, Thomas G, Beard D, Javaid MK, Murray D, Dieppe PA, Dreinhofer KE, Peter-Guenther K, Field R, Cooper C, Arden NK. Body Mass Index Is Not A Clinically Meaningful Predictor of Patient Reported Outcomes of Primary Hip Replacement Surgery: Prospective Cohort Study. Osteoarthritis and Cartilage. 2014;22(3): 431-439.

6. Konig G, Hamlin BR, Waters JH. Topical tranexamic acid reduces blood loss and transfusion rates in total hip and total knee arthroplasty. J Arthroplasty. 2013;28(9): 1473-1476.

7. Lemay E, Guay J, Cote C, Roy A. Tranexamic acid reduces the need for allogenic red blood cell transfusions in patients undergoing total hip replacement. Canadian Journal of Anasthesia. 2004;51(1): 31-37.

8. Martin JG, Cassatt KB, Kincaid-Cinnamon KA, Westendorf DS, Garton AS, Lemke JH. Topical Administration of Tranexamic Acid in Primary Total Hip and Total Knee Arthroplasty. J Arthroplasty. 2013.
9. Mayr E, Nogler M, Beneditti MG, Kessler O, Reinthaler A, Krismer M, Leardini A. A Prospective Randomized Assessment of Earlier functional Recovery in THA Patients Treated by Minimally Invasive Directed Anterior Approach: A Gait Analysis Study. Clinical Biomechanics. 2009;24(10): 812-818.

10. Morrison JJ, Dubose JJ, Rasmussen TE, Midwinter MJ. Military Application of Tranexamic Acid in Trauma Emergency Resuscitation (MATTERs) Study. Arch Surg. 2012;147(2): 113-119.

11. Saleh E, McClelland DB, Hay A, Semple D, Walsh TS. Prevalence of Anaemia Before Major Joint Arthroplasty and The Potential Impact of Preoperative Investigation and Correction on Perioperative Blood Transfusions. British Journal of Anaesthesia. 2007;99(6): 801-808.

12. Singh J, Ballal MS, Mitchell P, Denn PG. Effects of tranexamic acid on blood loss during total hip arthroplasy. Journal of Orthopaedic Surgery. 2010;18(3): 282286.

13. Sukeik M, Alshryda S, Haddad FS, Mason JM. Systematic review and meta-analysis of the use of tranexamic acid in total hip replacement. The Journal of Bone and Joint Surgery. 2011;93-B(1): 39- 46.

14. Tuttle JR, Ritterman SA, Cassidy DB, Anazonwu WA, Froehlich JA, Rubin LE. Cost Benefit Analysis of Topical Tranexamic Acid in Primary Total Hip and Knee Arthroplasty. J Arthroplasty. 2014.

15. Wind TC, Barfield WR, Moskal JT. The Effect of Tranexamic Acid on Transfusion Rate in Primary Total Hip Arthroplasty.” J Arthroplasty. 2013; 29(2): 387-389.

\section{Copyright \& Licensing}

Authors retain copyright and grant the journal right of first publication with the work. Reconstructive Review follows the Creative Commons Attribution-NonCommercial CC BY-NC. This license allows anyone to download works, build upon the material, and share them with others for non-commercial purposes as long as they credit the senior author, Reconstructive Review, and the Joint Implant Surgery \& Research Foundation (JISRF). An example credit would be: "Courtesy of (senior author's name), Reconstructive Review, JISRF, Chagrin Falls, Ohio". While works can be downloaded and shared they cannot be used commercially.

\section{JISRF Mission Statement}

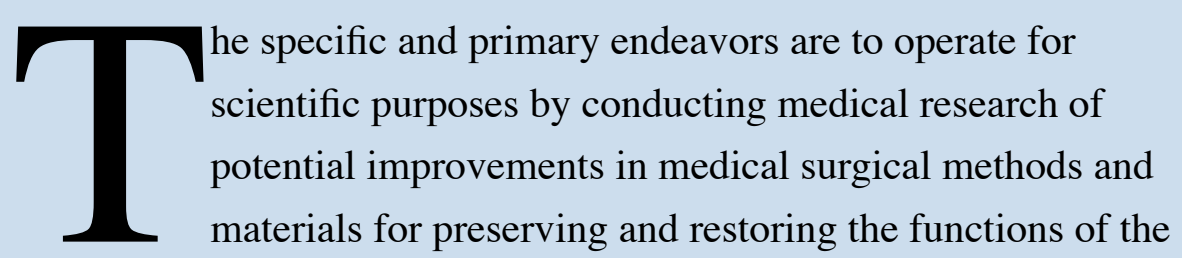

human body joints and associated structures which are threatened or

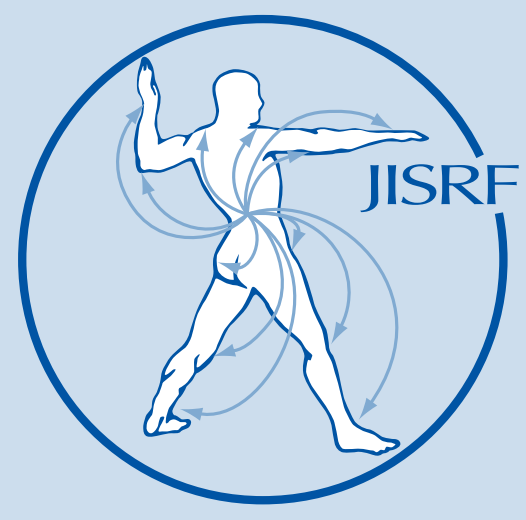

impaired by defects, lesions or diseases.

This Journal as all activities conducted by JISRF are available to all interested surgeons, scientists and educators. Our focus is on new cutting edge technologies, science - all with the intent to raise the level of discussion and discovery. Please become a part of this endeavor, we look forward to your interest and participation. 